\title{
DISTRIBUIÇÃO DIAMÉTRICA APÓS COLHEITA DE MADEIRAS COM ÊNFASE ÀS ESPÉCIES DE LECYTHIDACEAE
}

Pamella Carolline Marques dos Reis Reis ${ }^{1 *}$, Leonardo Pequeno Reis ${ }^{2}$, Agostinho Lopes de Souza ${ }^{3}$, Lyvia Julienne Sousa Rego ${ }^{4}$, Liniker Fernandes da Silva ${ }^{5}$

'Doutoranda do Programa de Pós-graduação em Ciência Florestal, Departamento de Engenharia Florestal, Universidade Federal de Viçosa, Viçosa, MG, Brasil. (pamella.reis@ufv.br)

${ }^{2}$ Doutorando do Programa de Pós-graduação em Ciência Florestal, Departamento de Engenharia Florestal, Universidade Federal de Viçosa, Viçosa, MG, Brasil.

${ }^{3}$ Professor do Programa de Pós-graduação em Ciência Florestal, Departamento de Engenharia Florestal, Universidade Federal de Viçosa, Viçosa, MG, Brasil.

${ }^{4}$ Doutoranda do Programa de Pós-graduação em Ciência Florestal, Departamento de Engenharia Florestal, Universidade Federal de Viçosa, Viçosa, MG, Brasil.

${ }^{5}$ Professor adjunto I da Universidade Federal do Recôncavo da Bahia, Cruz das Almas, BA, Brasil.

\section{Recebido em: 03/10/2016 - Aprovado em: 21/11/2016 - Publicado em: 05/12/2016 DOI: 10.18677/EnciBio_2016B_038}

\section{RESUMO}

No manejo florestal é necessário avaliar a dinâmica da estrutura diamétrica da comunidade e das espécies após a colheita seletiva no ciclo de corte para garantir a sustentabilidade da atividade. $O$ objetivo do trabalho foi avaliar a dinâmica da estrutura diamétrica de um grupo de espécies da família Lecythidaceae em uma área de colheita seletiva na Amazônia. A pesquisa foi realizada em uma floresta de 200 ha localizada em Moju-PA. Em 1997 foram colhidas na área $23 \mathrm{~m}^{3} \mathrm{ha}^{-1}$ de madeira de 25 espécies comerciais. No experimento foram aleatorizadas 22 parcelas permanentes com uma amostragem total de 11ha, e nessas foram mensuradas todas as árvores com DAP $\geq 10 \mathrm{~cm}$ em diferentes anos $(1995,1998 \mathrm{e}$ 2010). Não houve diferença a $5 \%$ de significância na estrutura diamétrica de Lecythidaceae nos períodos de 1995 a 1998 e 1998 a 2010, somente de 1995 a 2010. Essa diferença ocorreu por causa da diminuição do número de árvores nas primeiras classes de diâmetro após a colheita (13 anos), devido à mortalidade natural e passagem de árvores para as maiores classes de diâmetro, sendo esta saída não compensada pelo ingresso de novas árvores, apesar disso a forma de jinvertido foi mantida. As espécies de Couratari Aubl. e Lecythis pisonis Cambess que não apresentaram a forma contínua e decrescente (J-invertido), necessitam de um planejamento por espécie específico para garantir a sustentabilidade após a colheita e também são necessários estudos através de modelos matemáticos que expliquem o seu comportamento em relação a distribuição dos diâmetros.

PALAVRAS-CHAVE: Exploração florestal, manejo florestal, tauarí. 


\title{
DIAMETRIC DISTRIBUTION AFTER WOOD HARVEST WITH EMPHASIS ON LECYTHIDACEAE SPECIES
}

\begin{abstract}
In forest management it is necessary to evaluate the dynamics of diametric structure of community and species after selective harvest in the cutting cycle to ensure the sustainability of the activity. The aim of the study was to evaluate the dynamics of the diametric structure of a group of Lecythidaceae species in a selective harvest area in the Amazon. The research was conducted in a 200 ha forest located in Moju-PA. In 1997, $23 \mathrm{~m}^{3} \mathrm{ha}^{-1}$ of wood from 25 commercial species were harvested in the area. In the experiment 22 permanent plots were randomized with a total sample of 11 ha, on which all trees with DAP $\geq 10 \mathrm{~cm}$ in different years (1995, 1998 and 2010) were measured. There was no difference at $5 \%$ significance in the diametric structure of Lecythidaceae in the periods of 1995 to 1998 and 1998 to 2010, only from 1995 to 2010. This difference occurred due to the decrease in the number of trees in the first classes of diameter after harvest (13 years) due to natural mortality and trees passing to larger classes of diameter, and this output was not compensated by the inflow of new trees, despite that the inverted $\mathrm{J}$-shape was maintained. Couratari Aubl. and Lecythis pisonis Cambess species which did not have continuous and decreasing order (inverted $\mathrm{J}$ ), need a species-specific planning to ensure sustainability after harvest and also studies through mathematical models that explain their behavior in relation to distribution of diameters are necessary.
\end{abstract}

KEYWORDS: Forest Management, Tauarí, Forest Exploitation.

\section{INTRODUÇÃO}

A floresta Amazônica possui um elevado potencial econômico madeireiro. Há mais de 1 mil espécies de madeira na Amazônia, sendo que de 60 a 80 são consideradas comerciais (ADEODATO et al., 2011). Em 2009, foram extraídos 14,2 milhões de $\mathrm{m}^{3}$ de madeira em tora na Amazônia e o estado do Pará representou $46,6 \%$ desse total, com uma renda total de $R \$ 4,9$ bilhões (PEREIRA et al., 2010). Essa produção demonstra a importância da floresta Amazônica na geração de emprego e renda na região, mas a sua sustentabilidade depende da utilização do Manejo Florestal Sustentável embasado tecnicamente.

Para manejar a floresta é necessário conhecer a estrutura para que se obtenha a sustentabilidade ambiental ao longo prazo. Uma das principais estruturas que subsidia tecnicamente a colheita florestal e o potencial das espécies a serem colhidas é a distribuição dos diâmetros. Através da distribuição diamétrica é possível aferir sobre o histórico da espécie na área, a quantidade de árvores passíveis para a colheita, a regeneração natural e a sustentabilidade da espécie após a colheita. Também pode ser empregada em decisões técnicas de tratamentos silviculturais, como ocorre na técnica BDq na redução sistemática da área basal nas classes de diâmetro (WEAVER et al., 2009; HANSON et al., 2012).

Existem diversas famílias botânicas encontradas na Amazônia que apresentam elevado potêncial econômico e dentre estas destaca-se as espécies de Lecythidaceae, que tem importância econômica para a região. No estado do Pará, atualmente, 28 espécies de Lecythidaceae são colhidas para produção de madeira (REIS et al., 2014). Dentre essas espécies comerciais, sete foram avaliadas neste trabalho: Eschweilera coriacea (DC.) S.A. Mori, Eschweilera amazonica R. Knuth, Lecythis pisonis Cambess, Lecythis idatimon Aubl., Couratari stellata A.C. Sm, 
Couratari oblongifolia Ducke \& R. Knuth e Couratari guianensis Aubl (REIS et al., 2014).

Isso demonstra a importância do estudo da dinâmica da estrutura diamétrica destas espécies para se avaliar o impacto da colheita de madeiras na recuperação no ciclo de corte e garantir que não ocorra a superexploração (RICHARDSON \& PERES, 2016) de determinadas espécies por não apresentarem uma estrutura diamétrica que sustente a colheita. A superexploração nas classes ocorre quando ocorre intensa colheita desproporcional em determinadas classes, isso resulta em alterações na distribuição diamétrica e, consequentemente, demanda um longo período para a recuperação do estoque aproveitável das espécies colhidas (REIS et al., 2014).

O objetivo do trabalho foi avaliar a dinâmica da estrutura diamétrica de oito espécies de Lecythidaceae após a colheita seletiva de madeiras em floresta na Amazônia para subsidiar decisões silviculturais.

\section{MATERIAL E MÉTODOS}

O estudo foi realizado em uma área de floresta localizada no município de

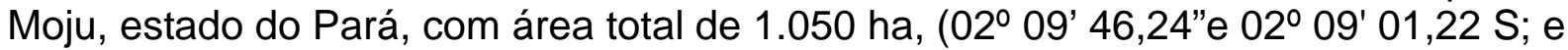
$48^{\circ} 47^{\prime} 33,94^{\prime \prime}$ e 48운 $48^{\prime} 02,95^{\prime \prime}$ W). O clima da região é do tipo Ami (quente e úmido), segundo a classificação de Köppen. A precipitação pluviométrica anual varia de 2.000 a $3.000 \mathrm{~mm}$. A umidade relativa do ar é de $85 \%$, com temperatura média anual de $26^{\circ} \mathrm{C}$. O relevo é plano, com formação de Latossolo Amarelo distrófico com diferentes texturas. A tipologia da área experimental é Floresta Ombrófila Densa de terra firme (REIS et al., 2013; REIS et al., 2015).

Em 1997 foi realizada uma colheita de madeiras em 200 ha, foram retiradas, em média, 3,3 árvores ha ${ }^{-1}$, correspondendo a um volume médio de $23 \mathrm{~m}^{3} \mathrm{ha}^{-1}$, de 25 espécies (REIS et al., 2015), considerando o Diâmetro Mínimo de Corte (DMC) de $65 \mathrm{~cm}$. Couratari guianensis foi a única espécie de Lecythidaceae que foi colhida, sendo colhido, em média, 0,21 árvores ha ${ }^{-1}$ com volume de $1,84 \mathrm{~m}^{3}$ ha ${ }^{-1}$ (REIS et al., 2014).

Em 1995, antes da colheita, foram implantadas, aleatoriamente, e medidas 22 parcelas permanentes com dimensões de $50 \times 100 \mathrm{~m}$, correspondendo a 11 ha de área amostral. Todas as árvores com Diâmetro à Altura do Peito (DAP) $\geq 10 \mathrm{~cm}$ foram registradas e medidas. As parcelas foram remedidas em 1998 e 2010 (REIS et al., 2015).

Para a identificação botânica das espécies, foram coletadas no mínimo cinco amostras de material botânico, por árvore, para cada nome vulgar de Lecythidaceae, totalizando 102 coletas. A determinação foi realizada por comparação com amostras botânicas existentes no herbário IAN da Embrapa (REIS et al., 2014).

Foi analisada a distribuição dos diâmetros das espécies de Lecythidaceae em diferentes períodos de 1995 a 1998, 1998 a 2010 e 1995 e 2010. A significância estatística para a distribuição diamétrica foi verificada pelo teste Qui-quadrado $\left(x^{2}\right)$ ao nível de $5 \%$ de significância entre os diferentes levantamentos. Agrupou-se as classes que apresentaram frequência menor que cinco árvores, usado a amplitude de $10 \mathrm{~cm}$, por ser mais usual em florestas tropicais na Amazônia. As análises estatísticas foram processadas pelo software Bioestat 5.3. 


\section{RESULTADOS E DISCUSSÃO}

A comunidade apresentou distribuição diamétrica contínua decrescente nas três ocasiões avaliadas (1995, 1998 e 2010), com maior quantidade de árvores nas classes inferiores de diâmetro, 15 e $25 \mathrm{~cm}$, e menor número nas últimas classes (Figura 1), seguindo a típica distribuição dos diâmetros em forma de J-invertido, que é comum às florestas inequiâneas tropicais (HIGUCHI et al., 2012; REIS et al., 2014; SOUZA et al., 2014). Essa distribuição tida como balanceada, segue uma tendência natural das florestas inequiâneas, o número de árvores em relação às classes de diâmetro decresce em uma progressão geométrica (SOUZA \& SOARES, 2013; REIS et al., 2014).

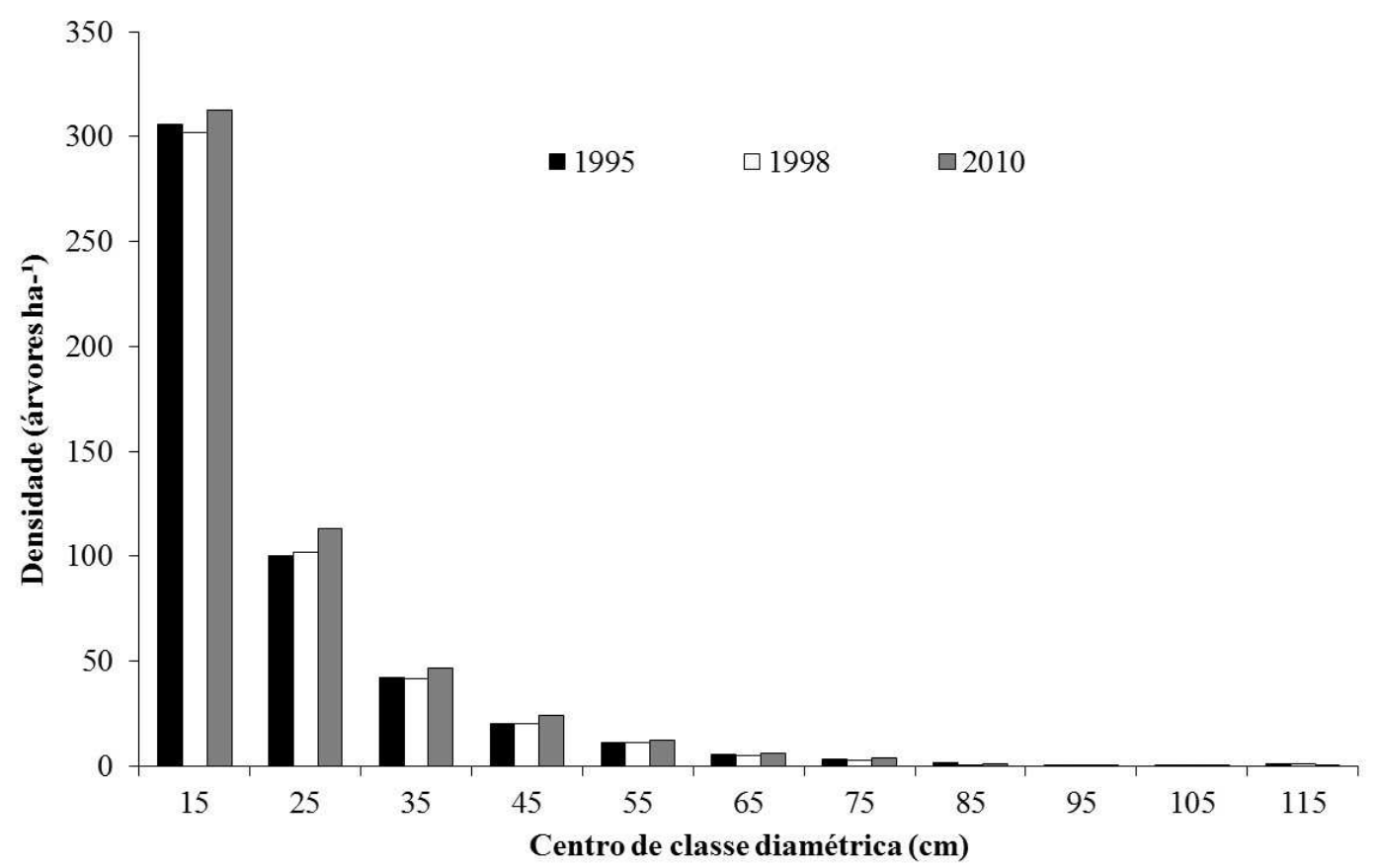

FIGURA 1. Distribuição diamétrica do total da comunidade em diferentes períodos: 1995, 1998 e 2010, em uma área colhida seletivamente no município de Moju-PA.

Em 1995, Lecythidaceae apresentava 98,7\% das árvores nas classes abaixo de $60 \mathrm{~cm}$ de diâmetro (Figura 2), com uma distribuição contínua e decrescente. Mesmo com a colheita em 1998, o padrão da distribuição diamétrica foi mantido, apresentando a mesma percentagem (98,7\%) de árvores nas classes abaixo de 60 $\mathrm{cm}$ de diâmetro. Em 2010 Lecythidaceae manteve-se com a distribuição de Jinvertido. Deve-se, este padrão, à capacidade de regeneração natural de muitas espécies das florestas tropicais (SOUZA et al., 2006).

Para toda a comunidade a distribuição diamétrica em todos os períodos analisados houve diferenças significativas: de 1995 a $1998\left(\mathrm{X}^{2}=28,778 ; \mathrm{Gl}=8 ; p<\right.$ $0,05), 1998$ a $2010\left(x^{2}=38,286 ; \mathrm{Gl}=8 ; p<0,05\right)$ e 1995 a $2010\left(x^{2}=40,452 ; \mathrm{Gl}=8 ; p\right.$ $<0,05)$. O total da Lecythidaceae não houve diferença significativa de 1995 a 1998 $\left(\mathrm{X}^{2}=2,394 ; \mathrm{Gl}=5 ; p>0,05\right)$ e 1998 a $2010\left(\mathrm{X}^{2}=6,752 ; \mathrm{Gl}=5 ; p>0,05\right)$, somente de 1995 a $2010\left(x^{2}=11,657 ; \mathrm{Gl}=5 ; p<0,05\right)$.

No primeiro momento após a colheita (1995 a 1998), o impacto da retirada das árvores não alterou a distribuição diamétrica da família, mas entre 1995 a 2010 
houve uma redução do número de árvores das duas primeiras classes de diâmetro, respectivamente, de 3,1 e 4,4\% (Figura 2), ocasionada pela mortalidade natural e saída de árvores por crescimento para as classes subsequentes. Demonstrando que o ingresso nas primeiras classes não foi suficiente para repor as perdas por mortalidade e movimentação no período. Também houve um aumento da densidade de $43,3 \%$ na classe de $45 \mathrm{~cm}$, causada pela entrada de árvores das classes menores nesta classe. Isso ocorre devido a aceleração do crescimento promovido pela abertura do dossel florestal após a colheita (SILVA et al., 2001; REIS et al. 2015).

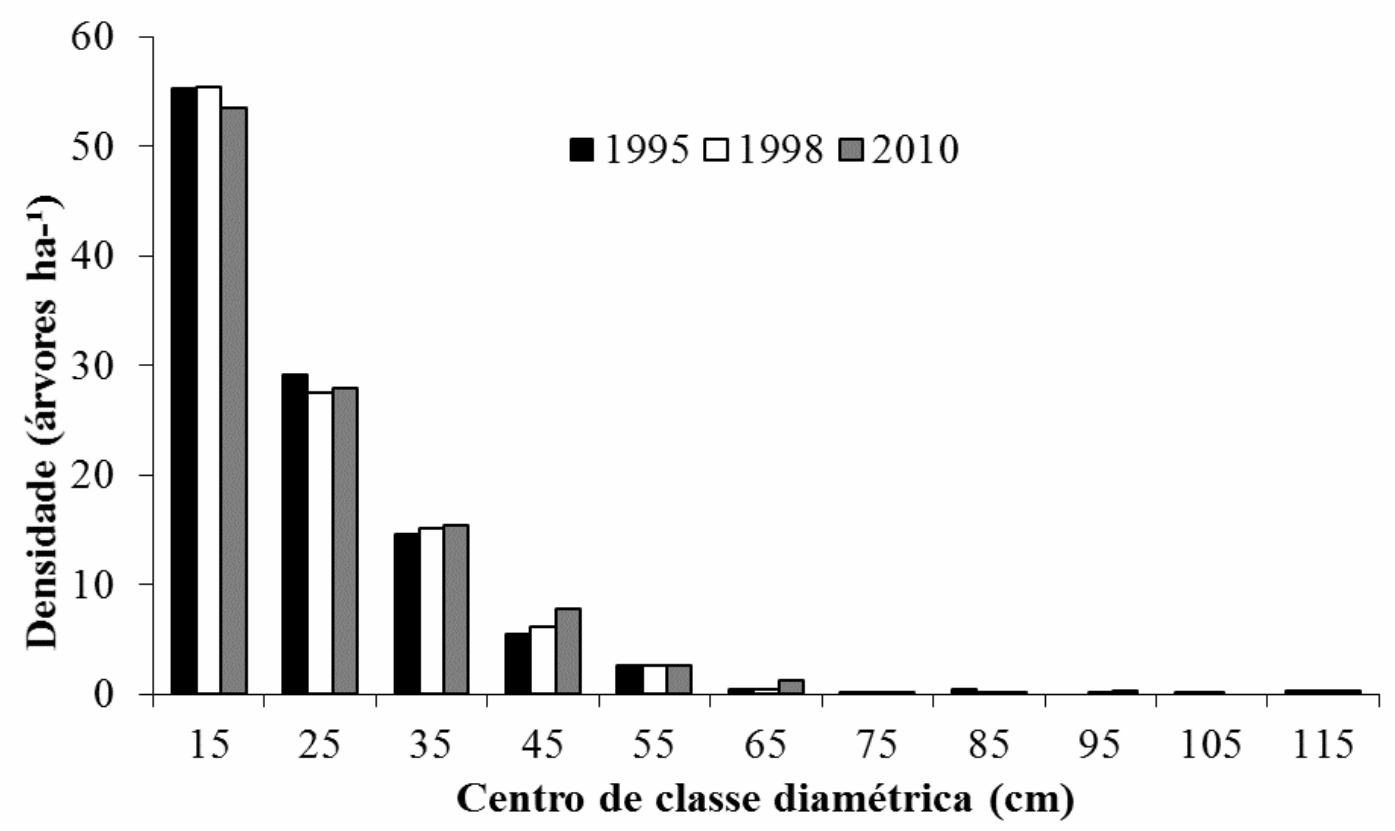

FIGURA 2. Distribuição dos diâmetros de Lecythidaceae antes (1995) e após a colheita (1998 e 2010) em uma área na Amazônia.

A distribuição diamétrica das espécies de Eschweilera com maior densidade, de acordo com REIS et al. (2014), E. coriacea, Eschweilera grandiflora (Aubl.) Sandwith e E. amazônica, tendem ao padrão J-invertido (Figura 3A, 3B e 3C). E. coriacea apresenta árvores em quase todas as classes de diâmetro nas três ocasiões observadas. Segundo REIS et al. (2014) esse comportamento indica estabilidade na recomposição florestal. Essas espécies se forem manejadas, terão capacidade de suportar e recuperar o estoque retirado na colheita. Nota-se que após a colheita (1998) a espécie $E$. coriacea apresentou uma diminuição das classes de 25 e $55 \mathrm{~cm}$. Entretanto, em 2010 observa-se a recuperação dessas classes (Figura $3 A)$.

Apesar de apresentarem árvores somente até a classe de $35 \mathrm{~cm}$ de DAP, as espécies E. grandiflora e E. amazonica, mantiveram esse padrão após a colheita, mostrando-se como espécies com distribuição diamétrica estável e dentro do padrão J-invertido. E. grandiflora apresentou um aumento no número de árvores em todas as classes de diâmetro após a colheita, caracterizando que se beneficiou com a mudança no dossel ocasionado pela colheita. O maior número de árvores nas classes inferiores, 49\% das árvores na classe de $15 \mathrm{~cm}$ em 2010, isso garante a sustentabilidade dessas espécies após a colheita por ter uma regeneração natural que irá recompor o estoque retirado no ciclo de corte. 

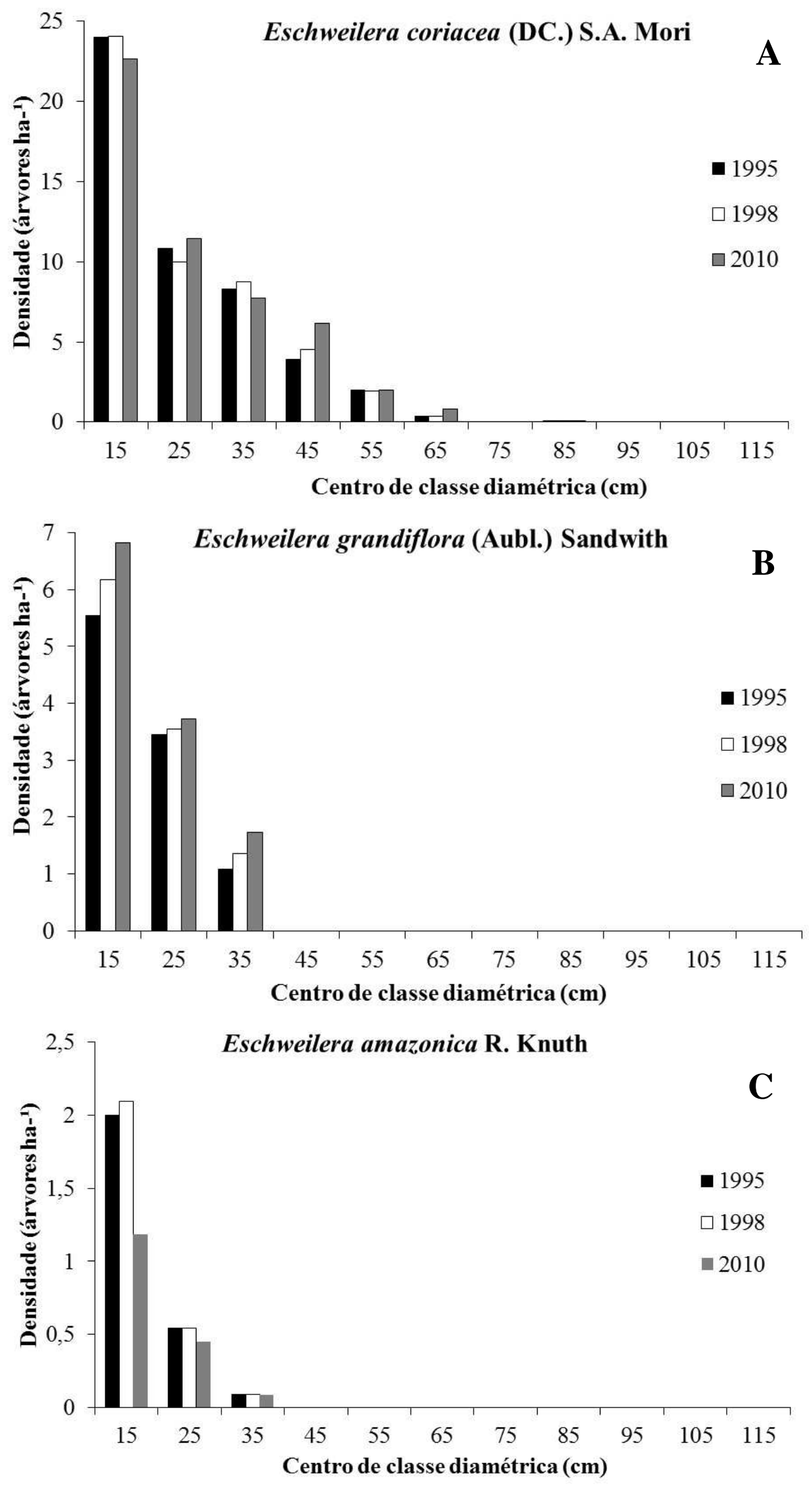


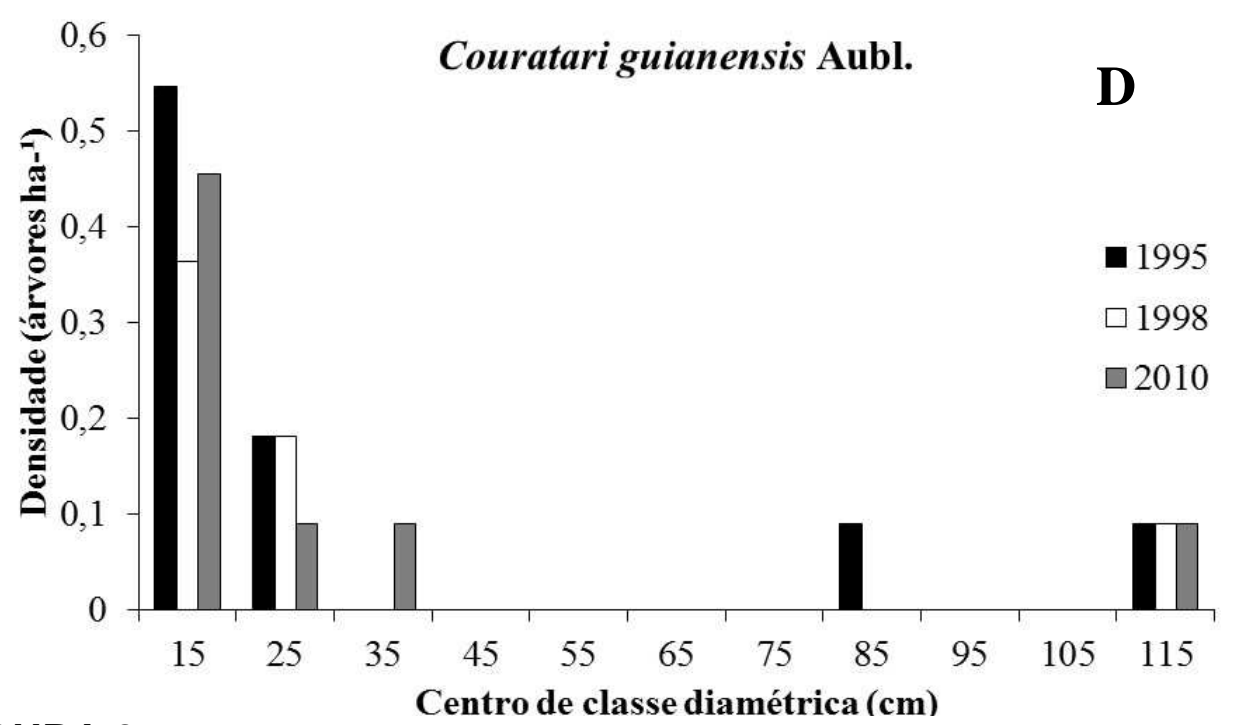

FIGURA 3. Distribuição dos diâmetros das espécies de Lecythidaceae: A - $E$. coreacea; B - E. grandiflora; C E. amazonica; D - C. guianensis. Em uma área colhida seletivamente na Amazônia.

A única espécie colhida de Lecythidaceae, C. guianensis, apresentou comportamento típico de espécies do grupo ecológico das tolerantes à sombra. Com maior número de árvores nas classes menores de diâmetro (15 e $25 \mathrm{~cm}$ ), diminuindo continuamente nas classes maiores. Após a colheita houve uma queda esperada no número de árvores na classe de $15 \mathrm{~cm}$ de diâmetro devido ao efeito da retirada de árvores, número esse que aumentou em 2010 devido ao recrutamento de árvores após a colheita. A classe de 25 cm se manteve estável de 1995 e 1998, com queda no número de árvores em 2010. Nota-se a existência de classes de diâmetro sem árvores (45 a $75 \mathrm{~cm}, 95 \mathrm{~cm}$ e $105 \mathrm{~cm}$ ) antes da colheita e 13 anos após. Isso foi considerado um evento altamente aleatório e muito observados em diversas espécies das florestas tropicais. Isso foi devido a mortalidade na classe vazia ou egresso na classe posterior.

A distribuição diamétrica de $C$. guianensis (Figura 3D) e C. stellata (Figura 3B) é típica de espécies tolerantes à sombra com a maior densidade nas menores classes de diâmetro, mas com certo desbalanceamento (algumas classes vazias). Distribuição semelhante da espécie também foi registrada na Amazônia ocidental por PRÓCÓPIO et al. (2010) e SILVA et al. (2008). C. oblongifolia (Figura 4A) apresentou a maioria dos indivíduos concentrados na classe de $45 \mathrm{~cm}$ e uma distribuição que se assemelha à de espécies intolerantes à sombra de longa vida: há indivíduos nas classes menores, classes intermediárias vazias e classes superiores com presença de árvores.

Estas árvores das classes superiores são aquelas que conseguiram atingir o dossel e se mantém na população por causa da menor competição por luz. Já as classes intermediárias vazias, com maior competição por luz, indicam que houve mortalidade devido a não abertura de clareiras para beneficiar o crescimento dos indivíduos até o dossel superior. De um modo geral as três espécies de Couratari apresentaram uma ou mais classes vazias. Há que se considerar, além da ecologia das espécies (FELFILI et al. 1998), a questão da intensidade amostral que pode ter sido insuficiente para gerar uma distribuição mais próxima da realidade das espécies. Espécies do gênero Couratari, por sua importância comercial, deveriam 
merecer atenção da pesquisa sobre sua ecologia para melhor orientar o planejamento de sua colheita.

Analisando as espécies de Lecythis com maior densidade, verificou-se que $L$. idatimon apresentou distribuição padrão J-invertido característico das espécies tolerantes e se mantém assim durante todo o período de monitoramento (Figura 4C). As reduções na densidade (provavelmente resultado do impacto da colheita) não afetaram sua estrutura diamétrica. FRANCEZ et al. (2009) também reportaram uma distribuição diamétrica semelhante para a espécie, em Paragominas-PA.
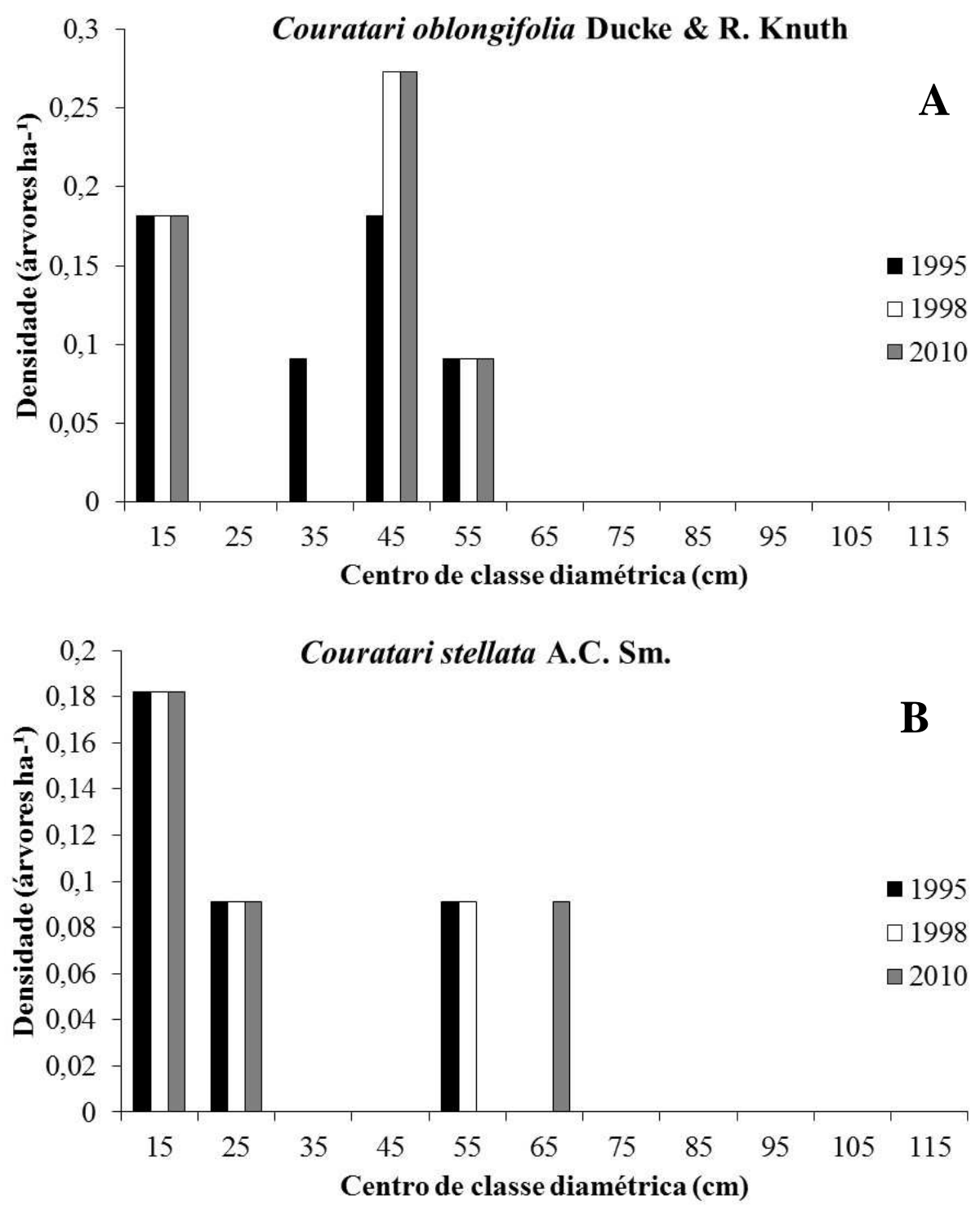

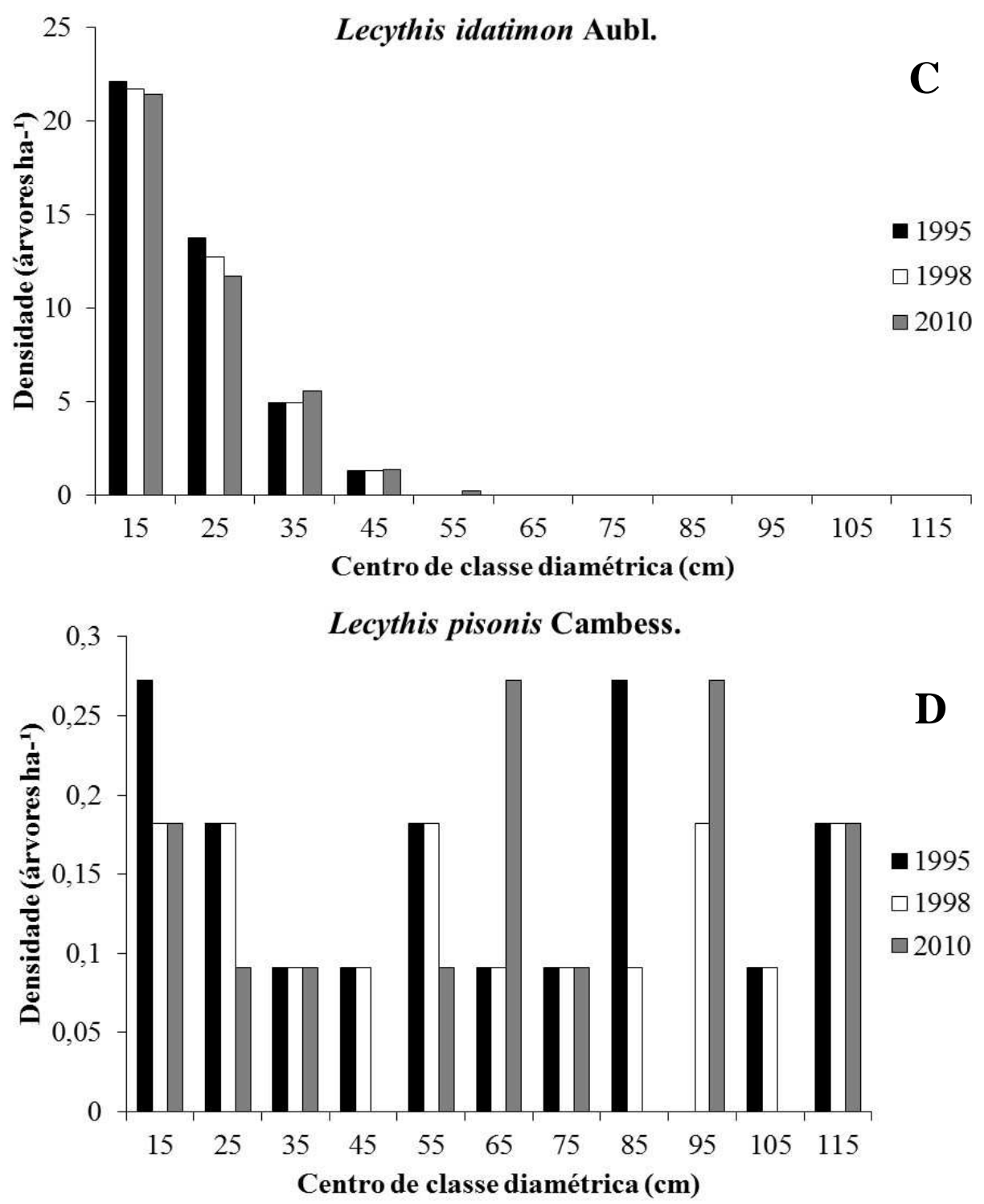

FIGURA 4. Distribuição dos diâmetros das espécies de Lecythidaceae: A C.oblongifolia; B - C. stellata; C - L. idatimon; D - L. pisonis. Em uma área colhida seletivamente na Amazônia.

A distribuição dos diâmetros mais diferenciada do padrão j-invertido foi a da $L$. pisonis (Figura 4D), com uma distribuição mais uniforme até mesmo antes da colheita. Tendo um número de árvores relativamente bem distribuída nas classes de diâmetro. Por sua distribuição singular, Lecythis pisonis e as espécies do gênero Couratari requerem estudos para descrever sua distribuição diamétrica através de modelos matemáticos, bem como sobre sua ecologia e dinâmica populacional. A colheita deve seguir um planejamento por espécie para se evitar superexploração de classes e se necessário a recomposição da regeneração via enriquecimento pós colheita. 


\section{CONCLUSÕES}

A estrutura diamétrica da família Lecythidaceae foi alterada 13 anos após a colheita de madeiras, apresentando redução nas menores classes de diâmetro. Mas manteve a forma de j-invertido.

Lecythis pisonis e as espécies do gênero Couratari não apresentaram a forma de j-invertido, caracterizando-as como espécies com um comportamento singular que necessitam de mais estudos no longo prazo após a colheita.

\section{AGRADECIMENTOS}

À Embrapa Amazônia Oriental pela concessão dos dados para a dissertação de mestrado que originou este artigo. À CAPES e ao CNPq pela concessão de bolsas de pós-graduação aos autores.

\section{REFERÊNCIAS}

ADEODATO, S.; MONZONI, M.; BETIOL, L. S.; MALU-VILLELA, M. Madeira de ponta a ponta: o caminho desde a floresta até o consumo. FGV ERA: São Paulo, 2011. p.128.

FELFILI, J. M. Determinação de padrões de distribuição de espécies em uma mata de galeria no Brasil Central com a utilização de técnicas de análise multivariada. Boletim do Herbário Ezechias Paulo Heringer 2: p.35-48, 1998.

FRANCEZ, L. M. B.; CARVALHO, J. O. P.; JARDIM, F. C. S.; QUANZ, B; PINHEIRO, K. A. O. Efeito de duas intensidades de colheita de madeira na estrutura de uma floresta natural na região de Paragominas, Pará. Acta Amazonica, v.39, n.4, p.851-864, 2009. Disponível em: < http://dx.doi.org/10.1590/S004459672009000400014>. doi: 10.1590/S0044-59672009000400014

HANSON, J. J.; LORIMER, C. G.; HALPIN, C. R.; PALIK, B. J. Ecological forestry in an uneven-aged, late-successional forest: Simulated effects of contrasting treatments on structure and yield. Forest Ecology and Management, v. 270, p. 94-107, 2012. Disponível em: <http://dx.doi.org/ 10.1016/j.foreco.2012.01.017>. doi: 10.1016/j.foreco.2012.01.017

HIGUCHI, F. G.; SIQUEIRA, J. D. P.; LIMA, A. J. N.; FIGUEIREDO FILHO, A.; HIGUCHI, N. Influência do tamanho da parcela na precisão da função de distribuição diamétrica de Weibull na floresta primária da Amazônia central. Floresta, v. 42, n. 3, p. 599-606, 2012. Disponível em: < http://dx.doi.org/10.5380/rf.v42i3.19640>. doi: 10.5380/rf.v42i3.19640

PEREIRA, D.; SANTOS, D.; VEDOVETO, M.; GUIMARÃES, J.; VERÍSSIMO, A. Fatos florestais da Amazônia 2010. Belém: Imazon, 2010. 124 p.

REIS, L. P.; RUSCHEL, A. R.; SILVA, J. N. M.; REIS, P. C. M.; CARVALHO, J. O. P.; SOARES, M. H. M. Dinâmica da distribuição diamétrica de algumas espécies de Sapotaceae após exploração florestal na Amazônia Oriental. Revista de Ciências Agrárias, v. $57, \quad$ n. 3 , p.234 - 243, 2014. Disponível em: < http://dx.doi.org/10.4322/rca.a01401>. doi: 10.4322/rca.ao1401 
REIS, L. P.; SILVA, J. N. M.; REIS, P. C. M.; CARVALHO, J. O. P.; QUEIROZ, W. T.; RUSCHEL, A. R. Efeito da exploração de impacto reduzido em algumas espécies de Sapotaceae no leste da Amazônia. Floresta, v. 43, n. 3, p.395 - 206, 2013. Disponível em: <http://dx.doi.org/10.5380/rf.v43i3.30808>. doi: 10.5380/rf.v43i3.30808

REIS, L. P; REIS, P. C. M.; RUSCHEL, A. R.; SILVA, J. N. M.; CARVALHO, J. O. P.; SOUZA, A. L.; SOARES, M. H. M.; MIYAHARA, R. K. N. Forest dynamics in the eastern Amazon with special reference to Sapotaceae species. Floresta, v. 45, n. 3, p. 567 - 576, 2015. Disponível em: <http://dx.doi.org/10.5380/rf.v45i3.35947>. doi: 10.5380/rf.v45i3.35947

REIS, P. C. M.; REIS, L. P.; RUSCHEL, A. R.; SILVA, J. N. M.; CARVALHO, J. O. P.; QUEIROZ, W. T. Effect of timber harvesting on density and basal area of Lecythidaceae species in the Eastern Amazon. Floresta, v. 44, n. 2, p. 229 - 238, 2014. Disponível em: <http://dx.doi.org/10.5380/rf.v44i2.33043>. doi: 10.5380/rf.v44i2.33043

RICHARDSON, V. A.; PERES, C. A. Temporal Decay in Timber Species Composition and Value in Amazonian Logging Concessions. PloS one, v. 11, n. 7, p. 1 - 22, 2016. Disponível em: <http://dx.doi.org/10.1371/journal.pone.0159035>. doi: 10.1371/journal.pone.0159035

SILVA, K. E.; MATOS, F. D. A.; FERREIRA, M. M. Composição florística e fitossociologia de espécies arbóreas do Parque Fenológico da Embrapa Amazônia Ocidental. Acta Amazonica, v. 38, n. 2, p. 213-222, 2008. Disponível em: <http://dx.doi.org/10.1590/S0044-59672008000200004>. doi: 10.1590/S004459672008000200004

SILVA, J. N. M.; SILVA, S. M. A. da; COSTA, D. H. M.; BAIMA, A. M. V.; OLIVEIRA, L. C. de; CARVALHO, J. O. P. de; LOPES, J. C. A. Crescimento, mortalidade e recrutamento em florestas de terra firme da Amazônia Oriental: observações nas regiões do Tapajós e Jarí. In: SILVA, J.N.M.; CARVALHO, J. O. P. de; YARED, J. A. G. (Eds.). A silvicultura na Amazônia Oriental: Contribuições do projeto Embrapa/DFID. Belém, 2001. p. 291-308.

SOUZA, C. R.; AZEVEDO, C. P.; ROSSI, L. B.; SANTOS, J.; HIGUCHI, N. Projection of diametric distribution and carbon stock of a managed forest in Manaus/AM. Floresta, v. 44, n. 3, p. 525-534, 2014. Disponível em: < http://dx.doi.org/10.5380/rf.v44i3.32854>. doi: 10.5380/rf.v44i3.32854

SOUZA, D. R.; SOUZA, A. L.; LEITE, H. G.; YARED, J. A. G. Análise estrutural em floresta ombrófila densa de terra firme não explorada, Amazônia Oriental. Revista Árvore, v. 30, n. 1, p. 75-87, 2006. Disponível em: < http://dx.doi.org/10.1590/S010067622006000100010>. doi: 10.1590/S0100-67622006000100010

SOUZA; A. L.; SOARES, C. P. B.; Florestas Nativas: estrutura, dinâmica e manejo. Viçosa: Ed. UFV, 2013. 322p. 
WEAVER, J. K.; KENEFIC, L; S.; SEYMOUR, R. S.; BRISSETTE, J. C. Decaying wood and tree regeneration in the Acadian Forest of Maine, USA. Forest Ecology and Management, v. 257, n. 7, p. 1623-1628, 2009. Disponível em: <http://dx.doi.org/10.1016/j.foreco.2009.01.023>. doi:101016/j.foreco.2009.01.023 